\title{
Erratum to: Stereoradiography imaging motion artifact: does it affect radiographic measures after spinal instrumentation?
}

\author{
Anne-Laure Simon ${ }^{1,2} \cdot$ Emmanuelle Ferrero $^{3}$ - A. N. Larson $^{4} \cdot$ Kenton R. Kaufman $^{1}$
}

Published online: 7 June 2017

(c) Springer-Verlag Berlin Heidelberg 2017

\section{Erratum to: Eur Spine J}

\section{DOI 10.1007/s00586-016-4462-z}

Unfortunately, the author, A. N. Larson name was wrongly displayed in the original publication. The correct name should read as A. N. Larson.

The original article has also been updated.

The online version of the original article can be found under doi: 10.1007/s00586-016-4462-z.

Kenton R. Kaufman

kaufman.kenton@mayo.edu

1 Motion Analysis Laboratory, Mayo Clinic, 10 Third Avenue N.W, Rochester, MN 55905, USA

2 Pediatric Orthopaedics Department, Robert Debré Hospital, 48 Bd Sérurier, 75019 Paris, France

3 Orthopaedic Surgery Department, Georges Pompidou Hospital, 20 Rue Leblanc, 75019 Paris, France

4 Pediatric Orthopaedics Department, Mayo Clinic, Gonda 14, 200 1st Street S.W, Rochester, MN 55905, USA 